\title{
SOME PHYSICAL ERRORS OF X-RAY TEXTURE MEASUREMENTS
}

\author{
YU. PERLOVICH \\ Moscow Engineering Physics Institute, Kashirskoe shosse 31, 115409 Moscow, \\ Russia
}

(Received 10 January 1995)

\begin{abstract}
Typical experimental situations of texture measurements are demonstrated involving failure to take into account some physical factors responsible for an inadequate texture description or imaginary texture changes. Among these factors there are inevitable texture inhomogeneities, inhomogeneous distribution of defects in deformed metal materials and resulting inhomogeneous lattice perfection by their heat treatment. It is shown that a formal approach to texture analysis does not allow to reveal a number of important regularities of structure formation.
\end{abstract}

KEY WORDS: Texture, inhomogeneity, distribution of defects, recovery, X-ray scattering capacity, substructure anisotropy, lattice perfection.

\section{INTRODUCTION}

A number of known physical factors exists in textured materials, which can decrease essentially correctness and efficiency of mathematical texture analysis. In particular, these factors should be taken into account before ODF calculation in order to avoid the situation, that the achieved precision of mathematical description would be higher than the accessible precision of experimental texture measurements. Among such factors there are:

1) texture inhomogeneity of any real material;

2) inevitable structure inhomogeneity of different texture components;

3) anisotropic character of substructure produced by many technological treatments.

In this paper some typical examples are presented to illustrate an influence of the mentioned factors on results of X-ray texture measurements in the form of direct pole figures obtained by usage of the standard procedures (Wasserman and Grewen 1962; Borodkina and Spector 1981).

Used abbreviation: DPF - direct pole figure; RD - rolling direction;

$$
\text { ND - normal direction; TD - transverse direction. }
$$




\section{TEXTURE INHOMOGENEITY}

A hierarchy of texture inhomogeneities, differing by areas of their manifestation, exists always in metal materials (Bunge 1982). An optimal practical classification of texture inhomogeneities should be based on the concrete possibilities of the used experimental method having its characteristic advantages and inevitable restrictions. Leaving aside the question about comparative merits of different methods, we shall consider a texture inhomogeneity as it can be seen by X-ray diffractometry. Though the obtained data testify about inadequate statistical significance of a single DPF, an interesting positive information relative to the sample prehistory and mechanisms of texture development is contained in these data also.

\section{Texture Inhomogeneity of Rolled Molybdenum Sheet}

Several identical samples were cut from the middle part of a cold-rolled sheet of alloyed molybdenum $0.1 \mathrm{~mm}$ thick and studied by use of the standard diffractometric procedure including measurement and construction of partial DPF $\{001\}$ with an angular radius $70^{\circ}$. By the used collimating slits the irradiated area of a sample comprised $50 \mathrm{~mm}^{2}$ and tended to increase as the tilt angle $\alpha$ increased, while the penetration depth of the used X-ray $\mathrm{Cu} \mathrm{K} \mathrm{K}_{\alpha}$ radiation was not more than $2 \mu \mathrm{m}$. An investigated volume, as large as this, is usual for diffractometric texture measurements. DPF $\{001\}$ for three neighbouring regions of the sheet are presented in Figure $1(\mathrm{a}-\mathrm{c})$. Though all shown DPF's contain the same sequence of pole density contours, configurations of these contours differ significantly, testifying about essential texture inhomogeneity of the sheet. When described by ideal orientations, texture differences of the studied samples reveal themselves even in lists of the main components. While predominance of the components (113-001-113)[110] is common for all samples, positions of other components in order of decreasing intensity change in passing from one sample to another:

$$
\begin{array}{ll}
\text { sample a } & -(111)[\overline{1} 2 \overline{1}],(111)[\overline{1} 10] \\
\text { sample b } & -(111)[\overline{1} 2 \overline{1}],(111)[1 \overline{2} 1],(111)[\overline{1} 10] \\
\text { sample c } & -(334)[\sim \overline{3} 4 \overline{1}],(111)[\overline{1} 10]
\end{array}
$$

The following technique was used to systematize the observed texture inhomogeneity. All maxima on diffractometric curves for different tilt angles $\alpha$ were marked, their coordinates $(\alpha, \beta)$ were determined and corresponding points were plotted in the stereographic projection. It turned out that points of maxima on succeeding concentric circles for all samples get to the same smooth contour with an accuracy of $2-3^{\circ}$ (Figure 1-d). This contour is a kind of DPF skeleton; both its form and position do not depend on the intensity ratio of different components in the texture of the concrete sample. It seems to be an invariant of the texture formation process in a given material under the used conditions. The analogous procedure, applied to the sheet of $0.3 \mathrm{~mm}$ thickness, i.e. cold-rolled by a smaller deformation degree, shows a rising spread $\left(5-7^{\circ}\right)$ of maxima points on concentric circles around the same contour. Analysis of the literature and own data shows that the final stable orientations of rolled Mo single crystals also get to this DPF skeleton. Some of them are marked by enumerated circles at the skeleton contour of maxima arrangement on Figure 1-d. 

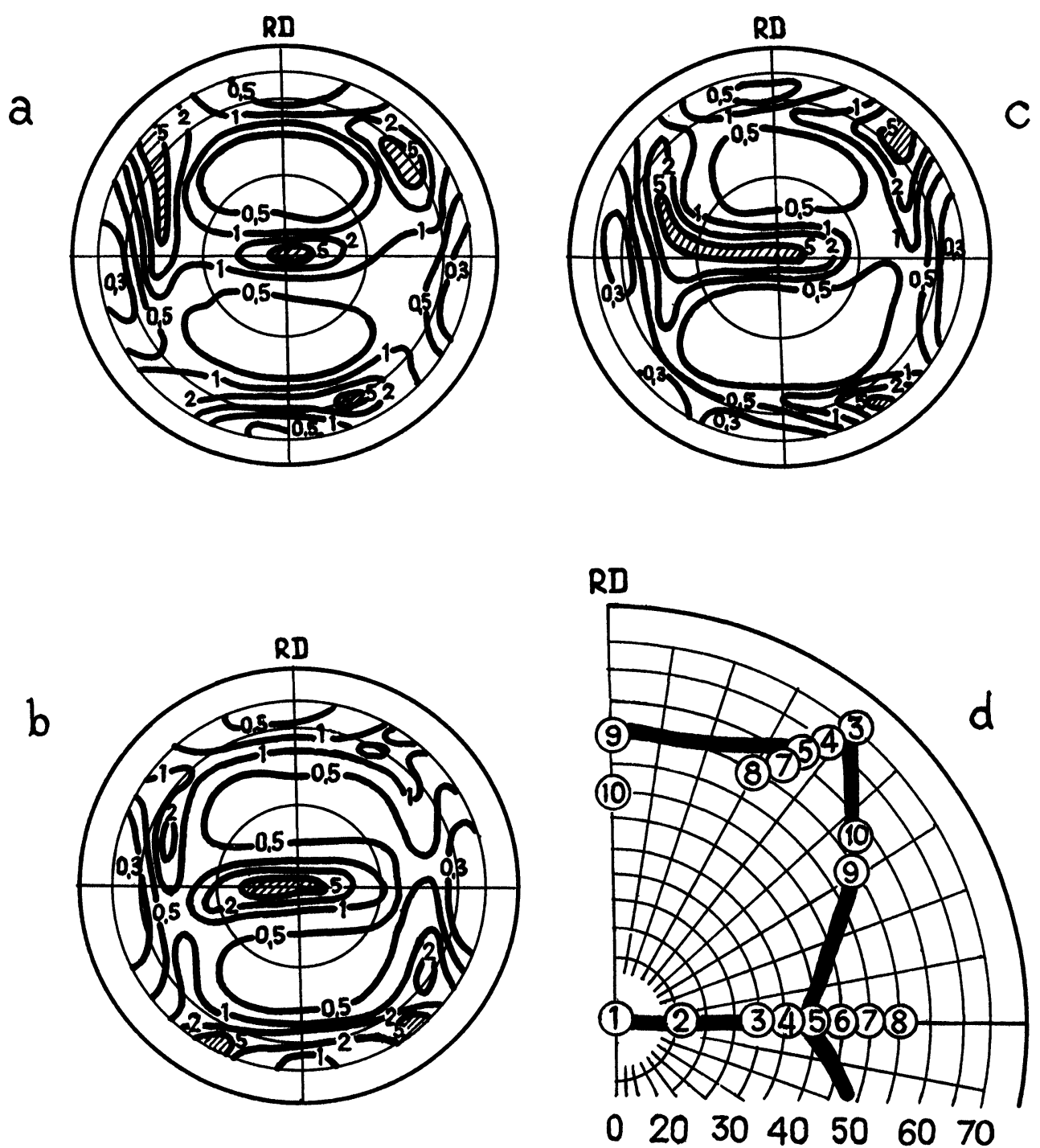

Figure 1 Texture inhomogeneity in the rolled sheet $(0.1 \mathrm{~mm}$ thick) of alloyed Mo:

$\mathrm{a}, \mathrm{b}, \mathrm{c}$ - partial DPF $\{001\}$ (angular radius $70^{\circ}$ ) for neighbouring regions from the central zone of the same sheet;

$\mathrm{d}$ - the "skeleton" contour of DPF $\{001\}$;

Numbers 1-10 within small circles correspond to final orientations of rolled Mo single crystals.

An origin of the observed texture inhomogeneity is clear: the final orientation of the rolled grain depends on its initial orientation. The coarser the grains in the initial workpiece, the more significant is the texture inhomogeneity of the cold-rolled sheet. In the considered case a number of deformed plate-like grains within an irradiated 
volume of the studied sample does not provide a sufficient statistic. Therefore it would be incorrect to judge the texture of the sheet (here we have not to do with some edge or surface effects!) by DPF measured for a single sample only. At the same time, existence of the invariant DPF skeleton, visible from a single measurement in spite of texture inhomogeneity, prompts new modes for effective treatment of texture data, including their mathematical treatment.

\section{Analysis of Texture Inhomogeneity in the Rolled Alloy $\mathrm{Zr}-2.5 \% \mathrm{Nb}$}

Another approach to systematization of texture inhomogeneities was elaborated as applied to a sheet of the alloy $\mathrm{Zr}-2.5 \% \mathrm{Nb}$. This alloy is used for production of construction elements for atomic reactors, and its texture inhomogeneity is of great practical importance. The main phase of the studied alloy is $\alpha$ - Zr having HCP crystalline lattice. Namely this phase determines an anisotropy of sheet properties, and changes of the basal normals distribution (0001) characterize most fully the process of texture development in $\alpha-\mathrm{Zr}$ (Douglass 1971).

The studied sheet of $0.2 \mathrm{~mm}$ thickness was produced by cold rolling with a total deformation degree of $60 \%$ transverse to the previous RD. Reorientation of $\alpha-\mathrm{Zr}$ grains connected with transverse rolling of the initial sheet is seen by comparison of DPF's (0001) presented in Figure 2. It should be taken into account that any registered DPF involves an occasional part originating not only from the real texture inhomogeneity, but from some inevitable experimental errors of the measuring procedure as well. Among these errors there are:

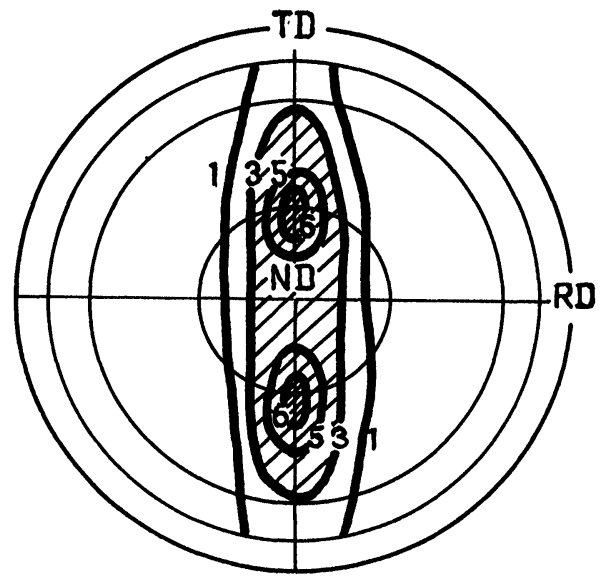

a

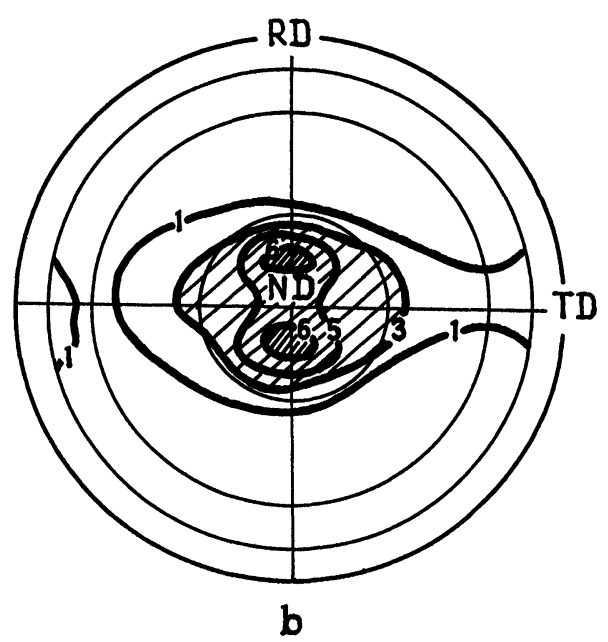

b

Figure 2 Texture formation in $\alpha-\mathrm{Zr}$ of $\mathrm{Zr}-2.5 \% \mathrm{Nb}$ alloy by cold rolling of the sheet. Partial DPFs $(0001)$ (angular radius $70^{\circ}$ ) were obtained by averaging of data for several samples from the initial sheet $0.5 \mathrm{~mm}$ thick (a) and from the final sheet $0.2 \mathrm{~mm}$ thick (b) rolled by $\varepsilon=60 \%$ transverse to the previous RD. 
$\sigma_{1}$ - an error due to the statistical nature of the X-ray quantum flux, being calculated as

$$
\alpha_{1}=\mathrm{I}^{-1 / 2}
$$

where I is the number of counted pulses;

$\sigma_{2}$ - an error due to inaccurate setting of the sample in initial and subsequent positions, being determined by results of multiple texture measurements for the same sample as a root-mean-square deviation from an average value I:

$$
\sigma_{2}=\left[\sum_{i=1}^{\mathrm{N}}\left(\mathrm{I}_{\mathrm{i}}-\overline{\mathrm{I}}\right)^{2} /(\mathrm{N}-1)\right] / \overline{\mathrm{I}}
$$

where $\mathrm{N}$ is the total number of identical measurements and $\mathrm{I}_{\mathrm{i}}$ is the result of $\mathrm{i}$-th measurement.

In Figure 3-a the distribution of $\sigma_{1}$ (on a percentage basis) is shown, having been calculated by the experimental data used for the construction of DPF (0001) (Figure 2-b). The character of this distribution fits the following principle: the lower is the pole density in a given point, the higher is the statistical error of registration for the corresponding sample position by texture measurement. The distribution of $\sigma_{2}$ (Figure 3-b) was constructed by results of 8 repeated identical texture measurements for the same sample. As for factors having an influence on the error $\sigma_{2}$, the most essential setting inaccuracy is connected with deviations of $\mathrm{RD}$ from a proper position owing to its uncontrolled rotation around ND. The angle of such rotation ranges up to $\pm 2^{\circ}$,
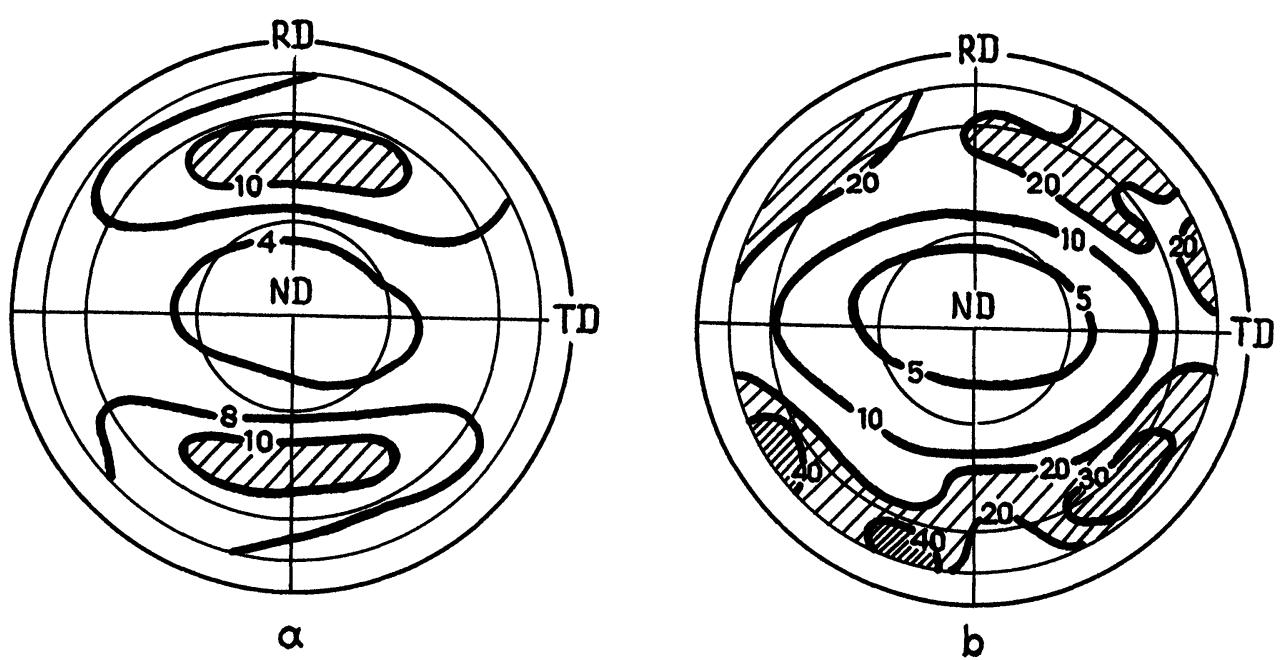

Figure 3 Experimental errors (on the percentage basis) of the measuring procedure for DPF (0001) of Figure 2-b.

a - distribution of the error $\sigma_{1}$ due to the statistical nature of the X-ray quantum flux;

$\mathrm{b}$ - distribution of the error $\sigma_{2}$ due to inaccurate setting of the sample. 
usually. So, on Figure 3-b zones of increased $\sigma_{2}$ show predominant circumferential stretching, whereas the highest values of $\sigma_{2}$ are attained within regions with the lowest pole density and its greatest local gradient, varying from $4 \%$ at texture maxima to $40 \%$ at texture minima.

To characterize a texture inhomogeneity of the sheet within the central area of its surface, 8 different samples were cut from this area and studied in identical conditions. The obtained experimental results were treated to construct a distribution of the rootmean-square deviation $\sigma_{3}$ (Figure 4-a) determined by analogy with $\sigma_{2}$ (Eq.2) with the only difference that in the case of $\sigma_{3}$ the values $I_{i}$ were obtained by measurements of different samples. For comparison the same procedure was used to describe the through-thickness texture inhomogeneity of the sheet. Seven layers of the same sample were studied successively after repeated electro-polishing. The corresponding root-meansquare deviation $\sigma_{4}$, definited according to Eq.2, is presented on Figure 4-b. But as opposed to samples used for study of the $\sigma_{3}$-distribution, successive layers used for the study of the $\sigma_{4}$-distribution contain some systematic texture variation because of different distances from the sheet surface. As a whole, obtained distributions of texture inhomogeneity (Figure 4-a,b) have a quite regular character, but at the same time they differ by positions of maxima both from DPF (Figure 2-b) and from the error distributions (Figure 3-a, b). This fact testifies that the distributions $\sigma_{3}$ and $\sigma_{4}$ reflect real texture inhomogeneities and that they can not be reduced to errors of measuring or setting.

The value of $\sigma_{3}$ varies from $10 \%$ to $40 \%$, and the value of $\sigma_{4}$ - from $10 \%$ to $30 \%$. Thus, in the considered case the texture inhomogeneity through sheet area is even more significant than through sheet thickness, and in addition to that, these two kinds of texture inhomogeneities differ by orientational regions of their manifestation. As a negative aspect of the presented results, it should be stated that in the general case texture measurement in a single sample is insufficient for full characterization of the
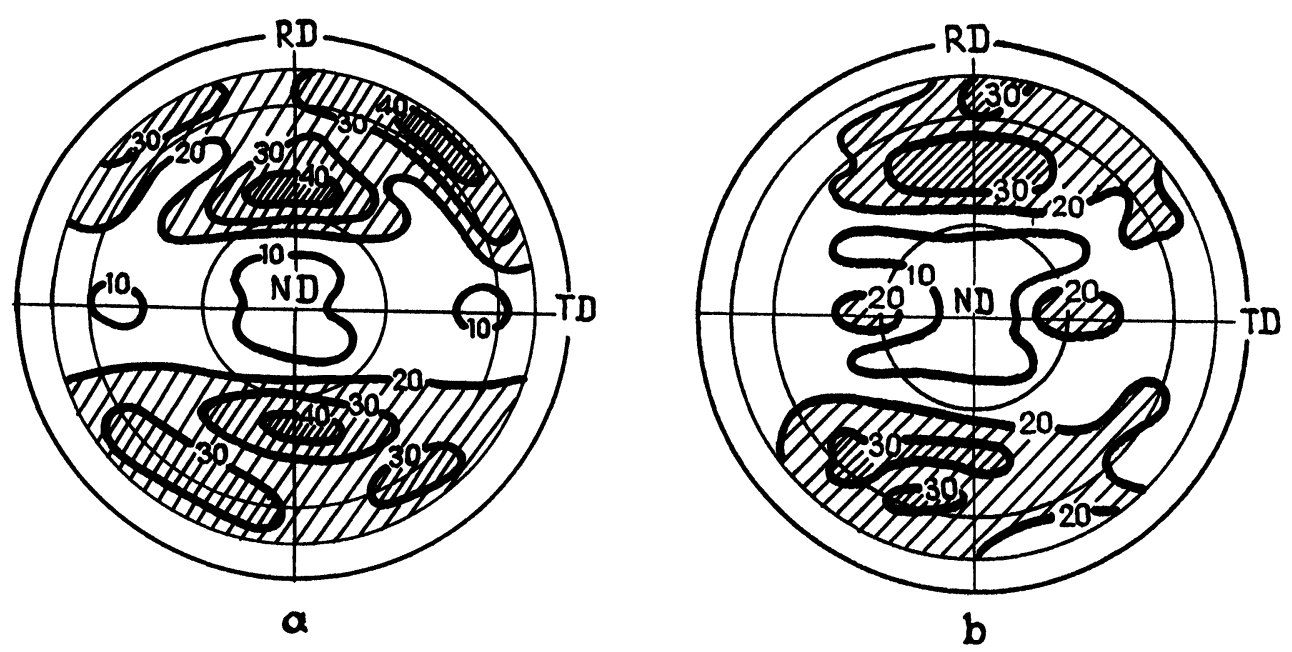

Figure 4 Texture inhomogeneity of $\alpha$-Zr over the surface (a) and across the thickness (b) of the studied sheet, characterized by distributions of relative root-mean-square deviations $\sigma_{3}$ and $\sigma_{4}$ from average values of measured intensity for DPF (0001). 
texture. In some contexts such insufficiency may be neglected, but at the same time many problems exist, which require the analysis of texture inhomogeneity before description of the results by ODF.

The informative content of the distributions $\sigma_{3}$ and $\sigma_{4}$ widens when considering the formation of the studied texture. Reorientation of $\alpha-\mathrm{Zr}$ grains in the course of rolling is caused by preferable action of different plastic deformation micromechanisms depending on the grain orientation. Among them there are three twinning systems as well as basal, pyramidal and prismatic slip or their combinations (Tenckhoff 1980). So, it is possible to associate the observed manifestations of texture inhomogeneity with the action of concrete mechanisms.

According to calculations (Macegorin, Rusakov and Evstyuchin 1980) and experimental data (Isaenkova and Perlovich $1987 \mathrm{a}, \mathrm{b}$ ) in the case of transverse rolling basal slip is the predominant mechanism of plastic deformation within grains of the main initial texture maxima (Figure 2-a). Namely basal slip is responsible for the observed shift of the maxima by $20^{\circ}$ towards the center of DPF (0001) (Figure 2-b), resulting in the formation of a quasi-stable texture with the main components $(0001) \pm 15-$ $25^{\circ} \mathrm{ND}-\mathrm{RD}<11 \overline{2} 1>$. The stability of this texture at intermediate deformation degrees is due to mutual equilibrium of basal and pyramidal slip. At the same time, accompanying changes of maxima configuration and scattering are connected with the action of different mechanisms in varying proportions. It is important that maxima of $\sigma_{3}$ and $\sigma_{4}$ are located just at the site of initial texture maxima removed by rolling owing to basal slip. Hence, the observed texture inhomogeneity reflects local differences of basal slip activation within grains incorporated in the original texture maxima.

Ambiguous realization of texture formation regularities being marked by an increase of $\sigma_{3}$ value testifies that activation of predicted mechanisms in grains of the given orientation is embarrassed to some degree. Really, the maximum of $\sigma_{3}$ is situated at the orientational zone, where acting shear stresses are low for most deformation systems except basal slip. But the critical shear stress for basal slip is relatively high, and therefore an uncertainty in the behaviour of the corresponding $\alpha$-grains can arise being conditioned by some additional minor factors. In particular, it seems likely that the initial distributions of grain size and grain misorientation within an irradiated area of several square millimeters, varying when passing from one sample to another, have an effect on the activation of basal slip and on the resulting reorientation of grains from the region of DPF (0001) with increased $\sigma_{3}$, where these grains were located initially.

As to texture inhomogeneity of the sheet across its thickness, the $\sigma_{4}$-distribution repeats the most features of the $\sigma_{3}$-distribution and has also additional maxima at the sites, where the final components $(0001) \pm 30-40^{\circ} \mathrm{ND}-\mathrm{TD}<10 \overline{1} 0>$ of the $\alpha-\mathrm{Zr}$ rolling texture develop (Figure 4-b), being stable to mutual equilibrium of prismatic and basal slip by retardation of slip in pyramidal systems. So, successive layers of the sheet differ by development of these final texture components. According to the theory of texture formation (Wassermann and Grewen 1962), the stability of final texture components is conditioned by the balanced action of different slip systems. Therefore, the observed texture inhomogeneity is connected with some variation of local conditions for such a balance. Various concrete structure-sensitive factors can be implied by such local conditions. For example, if action of different slip systems depends on substructure parameters by their own manners, minor substructure variations by passing from one layer to another would influence the probability of mutual equilibrium between acting systems, and thereby - the intensity of texture components considered. 


\section{INHOMOGENEOUS DISTRIBUTION OF DEFECTS IN DEFORMED GRAINS WITH DIFFERENT ORIENTATIONS}

Systematic investigations by use of special X-ray methods have shown that the distribution of strain hardening in rolled textured metal materials, especially with BCC lattice, has very inhomogeneous, though regular, character (Perlovich 1983, 1994). In particular, in rolled BCC metals, grains of the main texture component $\{001\}<011>$ exhibit significantly lower distortion of crystalline lattice, than grains of other main components with $\{111\}$ parallel to the rolling plane. Besides, lattice distortion because of plastic deformation increases as the grain orientation moves from a texture maximum to a neighbouring texture minimum. These facts are consequences of the regular dependence between an actual combination of active slip systems and the grain orientation. Since each micromechanism of plastic deformation has influence in its own manner on the grain substructure in the form of a characteristics lattice distortion, inhomogeneous conditions for deformation development are responsible for inhomogeneous strain hardening.

The considered lattice distortion involves an increased content of various defects, including point and linear ones. According to the theory of X-ray scattering in real crystals (Krivoglaz 1967), point and linear defects fall into different types with respect to their influence on parameters of X-rays diffraction. Dislocations cause widening of $\mathrm{X}$-ray lines by conservation of their integral intensity. Point defects, in contrast with dislocations, reduce the intensity of X-ray lines, having no influence on their widths. Since the standard texture measuring procedure foresees usage of wide intake slits at the detector in order to register the whole X-ray line independently of its width, this procedure should be insensitive to the content of dislocation within the textured sample in cases of small and even middle Bragg angles, being always inevitabely influenced by the content of point defects. When the Bragg angle of a registered X-ray line is large, long "tails" of this line can remain outside of the intake slit, and then the content of dislocations contributes to a decrease of the measured intensity, too. Since grains of deformed textured polycrystals differ by the content of defects depending on grain orientations, the resulting decrease of registered intensity is inhomogeneous. As a consequence, measured intensities of the same X-ray reflection for grains with different orientations are not proportional to the volume fractions of these grains, contrary to the main principle of X-ray texture analysis.

It was to be expected that annealing of a deformed sample at the temperature of recovery would cause the reverse phenomena - an inhomogeneous decrease of defect density, resulting in the corresponding inhomogeneous increase of X-ray scattering capacity and registered intensity as well as some imaginary changes of deformation texture.

The validity of this suggestion was confirmed as applied to the cold-rolled alloyed molybdenum. Temperatures of annealing $\left(200-400^{\circ} \mathrm{C}\right)$ were chosen in such a manner as to have conditions for the early stage of recovery, that is to reduce the content of point defects preferably. The recrystallization temperature for the studied alloyed molybdenum exceeds $1000^{\circ} \mathrm{C}$; so, in this material, at $200-400^{\circ} \mathrm{C}$ no texture changes due to recrystallization are possible. Nevertheless, when comparing results of texture measurements for the same sample after cold rolling by a deformation degree of $70 \%$ (Figure 5-a) and after following annealing at $300^{\circ} \mathrm{C}$ (Figure 5-b), essential texture changes are observed being explained actually by inhomogeneous recovery. 

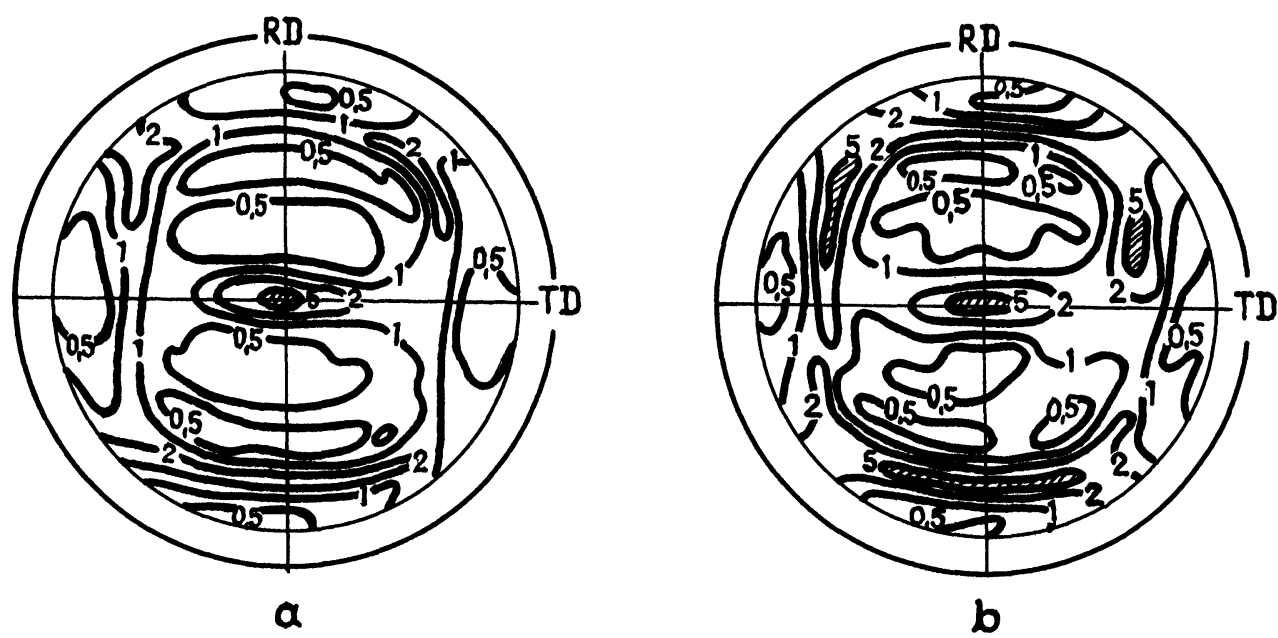

Figure 5 Influence of recovery on the appearance of DPF $\{001\}$ of the sheet sample of the alloyed molybdenum: a - cold rolled, $\varepsilon=70 \%$; b - annealed $300^{\circ} \mathrm{C}, 165 \mathrm{~min}$.

Repeated multiple measurements of DPF $\{001\}$ for the same sample were carried out interspersed with short annealings at recovery temperatures $\left(200^{\circ}, 300^{\circ}\right.$ and $\left.400^{\circ} \mathrm{C}\right)$. For measurement of DPF $\{001\}$ the X-ray reflection $(004)_{\mathrm{Cu}}$ with the angle $2 \theta=156^{\circ}$ was used having a wide profile and long "tails", therefore, the observed intensity changes are connected with a decrease of contents of both point defects and dislocations. A special attention was given to keep the setting of the sample constant and to use the same regime of texture measurements after each subsequent annealing. For each point $(\alpha, \beta)$ the curve of relative intensity increase $\mathrm{I} / \mathrm{I}_{0}$ versus annealing duration $\tau$ was constructed. In Figure 6 such curves for different characteristic points of DPF $\{001\}$ are shown. It is seen that for the same duration of annealing the ratio $\mathrm{I} / \mathrm{I}_{\mathrm{o}}$ can vary by two times depending on $(\alpha, \beta)$. As a result, an imaginary growth of pole density takes place in those points of DPF $\{001\}$, where the recovery processed were most vigorous. It should be noted that within texture minima the registered intensity increase $\mathrm{I} / \mathrm{I}_{\mathrm{o}}$ ranges up to $4-5$, reaching its peak. However, the precision of intensity measurements within texture minima is too low for constructing of reliable quantitative diagrams. Therefore, for the description of recovery in grains of texture minima we restrict ourselves to revealing of a qualitative tendency.

Using point-by-point comparison of pole figures, measured for the same sample after rolling and after annealings, distributions of intensity increase for different stages of recovery were constructed in the stereographic projection. Depending on the context, the terms "diagram of recovery inhomogeneity" or PF $\left(\mathrm{I} / \mathrm{I}_{0}\right)_{004}$ are used in reference to this distributions. Contours of $P F\left(I / I_{0}\right)_{004}$ pass through points of equal values $I / I_{0}$. In Figure $7 \mathrm{DPF}\{001\}$ (grey contours) and $\mathrm{PF}\left(\mathrm{I} / \mathrm{I}_{0}\right)_{004}$ (black contours) are shown together for recovery annealings at $300^{\circ} \mathrm{C}, 165 \mathrm{~min}$ (a) and $400^{\circ} \mathrm{C}, 70 \mathrm{~min}$ (b). Maxima of the $\mathrm{I} / \mathrm{I}_{\mathrm{o}}$-distributions are situated at "slopes" of texture maxima, just as abovementioned maxima of strain hardening. Thus, as might be expected, inhomogeneity of recovery is similar to inhomogeneity of strain hardening. 


\section{$R D$}
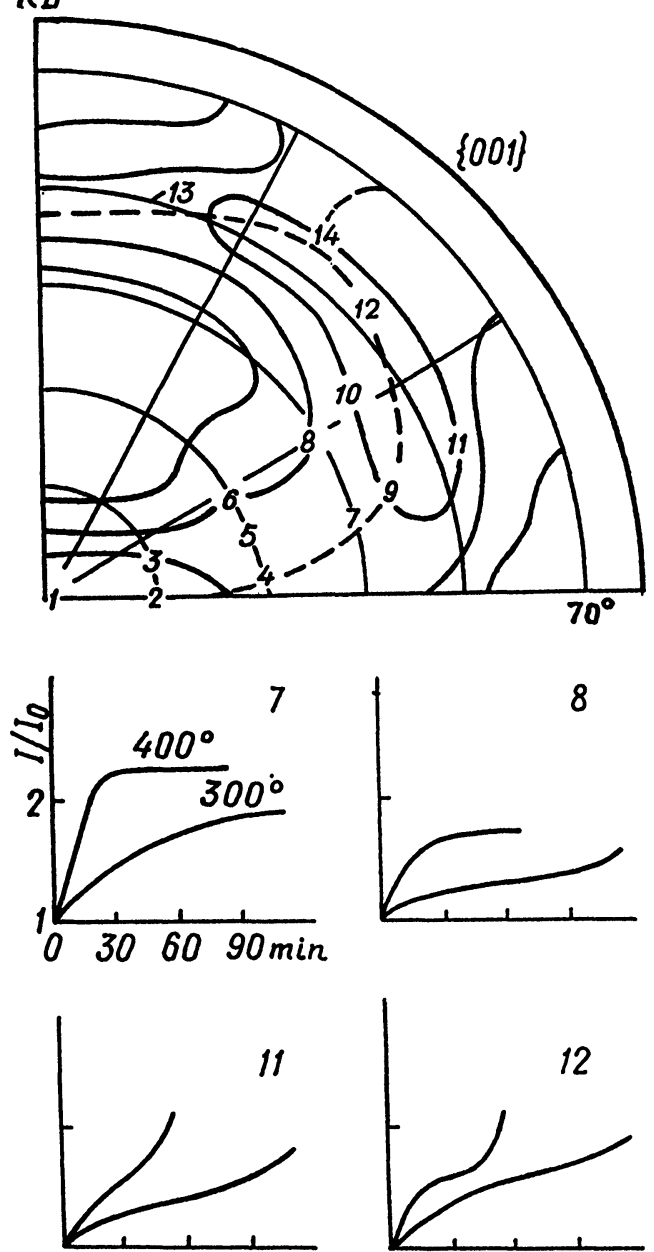
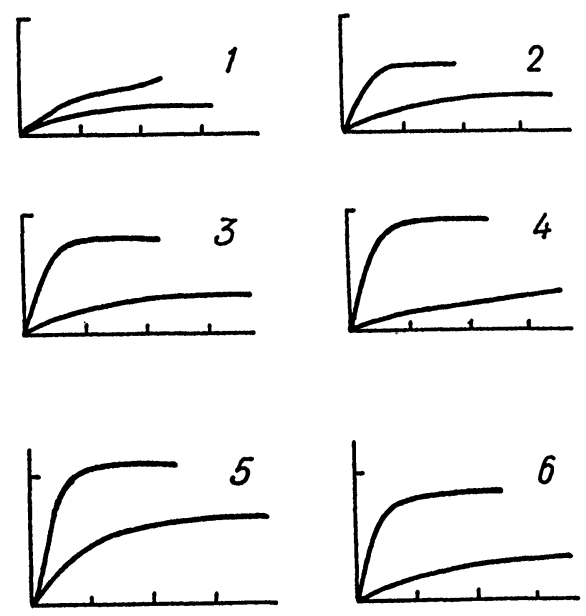

9
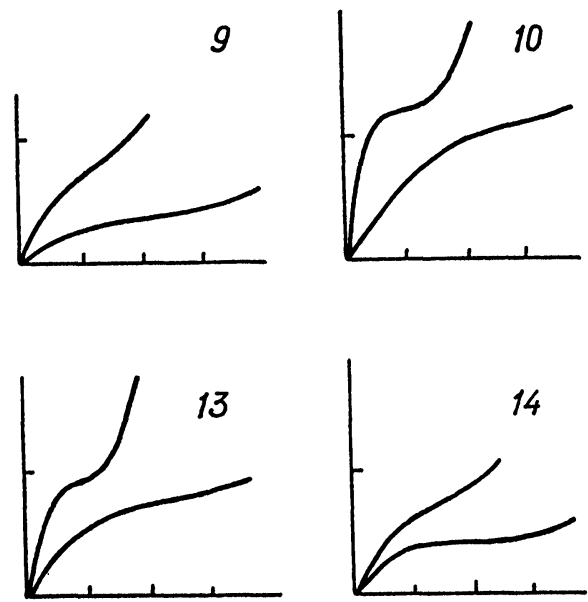

Figure 6 Curves of intensity changes for X-ray line (004) depending on the duration of isothermal annealing at $300^{\circ}$ and $400^{\circ} \mathrm{C}$ for grains with different orientations of crystallographic normals $<001>$. Each plot refers to a marked point of DPF $\{001\}$ having the corresponding number.

Figure 8 allows to compare in detail recovery processes in grains corresponding to the main texture components $\{001\}<011>$ and $\{111\}<112>$ respectively. Whereas recovery at $200^{\circ} \mathrm{C}$ shows a single stage only, recovery at $300^{\circ} \mathrm{C}$ or at higher temperatures exhibits two stages, at least for some grain orientations and, in particular, for the orientation $\{111\}<112>$. When using the method of sections (Damask and Diens 1963), activation energies for elementary recovery processes were calculated for both observed stages. For the first stage this energy is equal to $0.65-0.8 \mathrm{eV}\left(\mathrm{E}_{1}\right)$, and for the second stage - 1.1-1.2 eV $\left(\mathrm{E}_{2}\right)$. The calculated values of the activation energy testify that recovery proceeds at first due to migration of vacancies, and when the content of point 


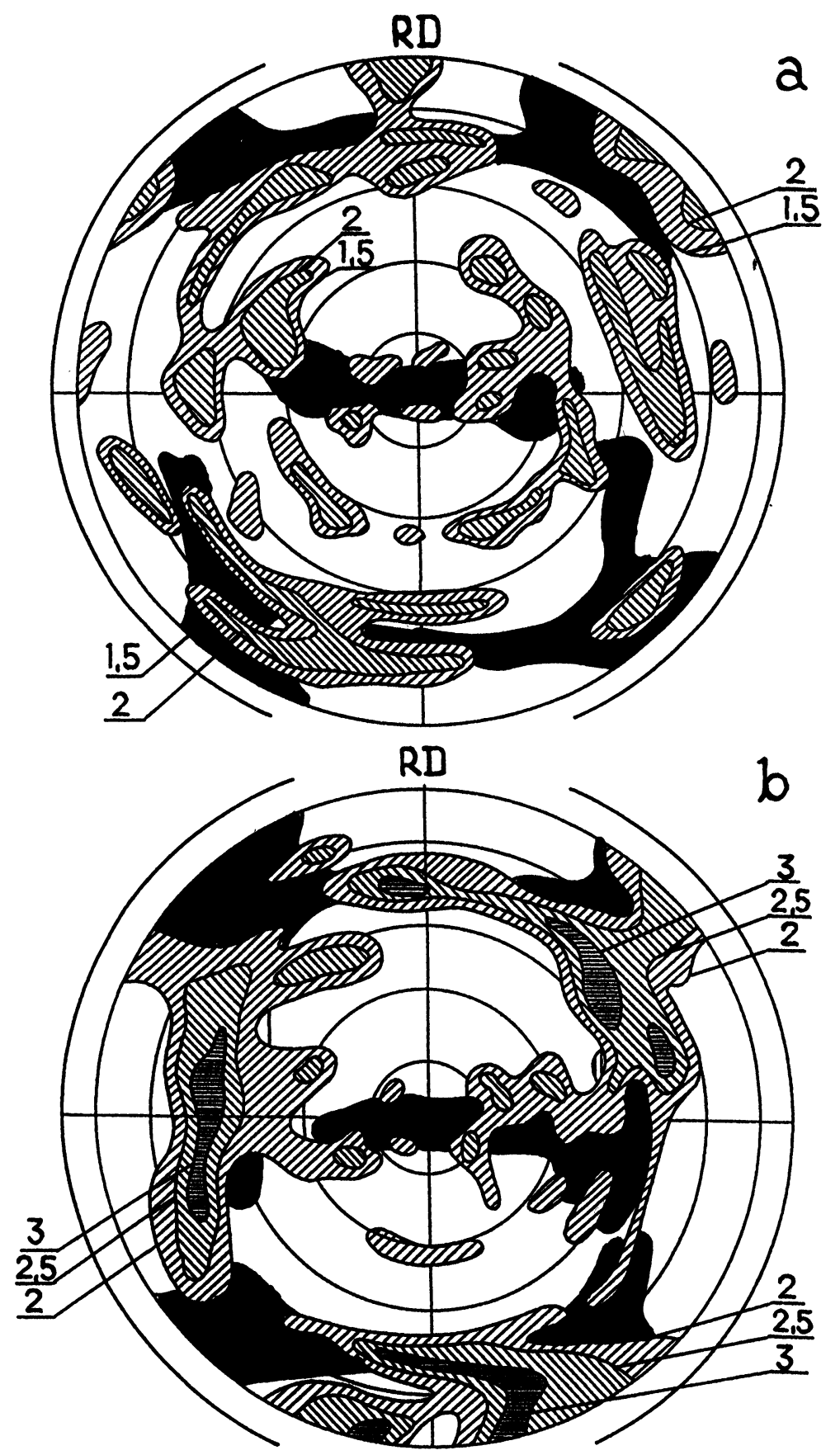

Figure 7 Diagrams of recovery inhomogeneity:

a $-300^{\circ} \mathrm{C}, 165 \mathrm{~min} ; \mathrm{b}-400^{\circ} \mathrm{C}, 70 \mathrm{~min}$.

Lower (blackened) contours - DPF $\{001\}$; upper contours - PF $\left(\mathrm{I} / \mathrm{I}_{0}\right)_{004}$. 


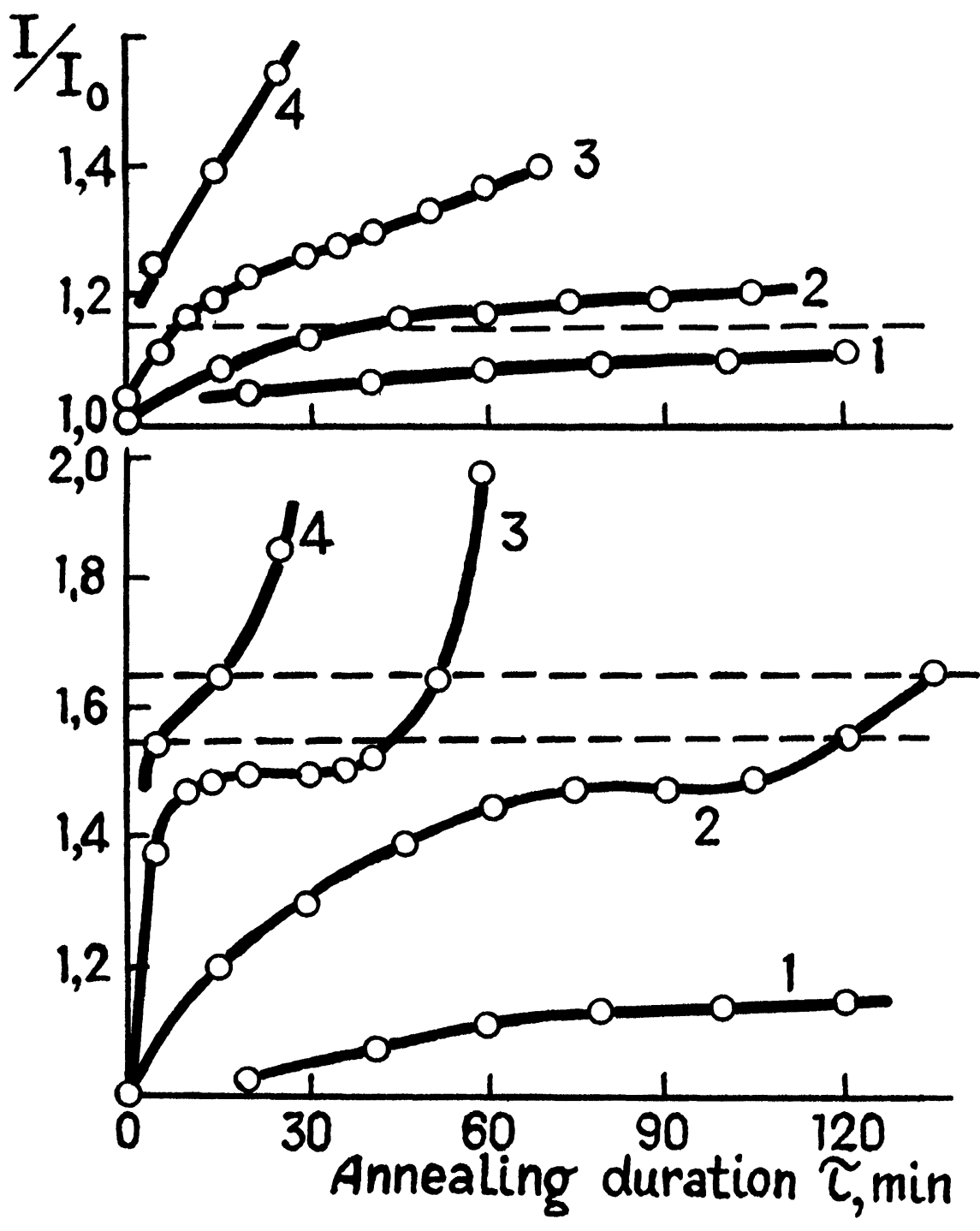

Figure 8 To evaluation of the activation energy for elementary recovery processes in grain orientations $\{001\}<011>$ (a) and $\{111\}<112>$ (b). Numbers of curves correspond to the following annealing temperatures: 1) $200^{\circ} \mathrm{C}$, 2) $300^{\circ} \mathrm{C}$, 3) $400^{\circ} \mathrm{C}$, 4) $500^{\circ} \mathrm{C}$. Used sections are shown by dashed lines.

defects occurs to be sufficiently low, redistribution of dislocations (with the activation energy $E_{2}$ ) controls the further development of recovery. During the second stage of recovery, the difference between the reflecting capacities of grains with orientations $\{111\}<112>$ and $\{001\}<011>$ increases additionally. If the inhomogeneous character 
of recovery would not be taken into account, the presented results of repeated texture measurements would be interpreted as an evidence in favour of 1.3-1.5 times growth of the components $\{111\}<112>$ at the expense of the component $\{001\}<011>$. However, such mutual absorbtion of grains with different orientations is impossible at the used annealing temperatures. Therefore, nothing more than an inhomogeneous decrease of the defect content takes place in the rolled sheet of alloyed molybdenum after annealing instead of supposed texture changes.

The observed effect of inhomogeneous reflecting capacity is too strong in order top be neglected by quantitative texture description. And an important conclusion follows from the above-presented results: in actual practice a measured DPF of deformed material is some superposition of real texture data with inhomogeneous distribution of X-ray reflecting capacity. Recovery leads to equalization of the reflecting capacities over grains of all orientations, - so, in consequence of recovery, an influence of inhomogeneous reflecting capacity on results of X-ray texture measurements decreases. Hence, in order to obtain the true data about deformation texture in the rolled sheet of alloyed molybdenum, the sample should be annealed at the recovery temperature. Otherwise both experimental DPF's and calculated ODF would contain some physical errors higher than those of the used mathematical ODF procedure.

Of course, this effect should be taken into account only in reference to those metal materials, which experience recovery at relatively high temperatures. Since in aluminium alloys, for example, recovery develops at room temperature already, errors of the considered type are avoided by X-ray study of their deformation textures. At the same time, the majority of deformed BCC metal materials shows necessity to correct experimental texture data with regard to inhomogeneous distribution of defects in textured polycrystals and to the corresponding inhomogeneity of X-ray reflecting capacity.

\section{MUTUAL INEQUALITY OF TEXTURE MAXIMA BELONGING TO THE SAME COMPONENT AS A RESULT OF SUBSTRUCTURE ANISOTROPY}

As practice of texture analysis shows, measured pole figures undergo strong distortions owing to substructure anisotropy of rolled metal materials. Generally accepted models of X-ray diffraction (Warren 1969) show that the shapes of X-ray lines characterize the condition of the crystal lattice along the normal to reflecting crystallographic planes $\{h k l\}$. Different families of these planes within grains of the same main orientation have projections at different regions of DPF contributing to different texture maxima of the same component. Normals from the same set $<\mathrm{hkl}>$ intersect grains of the textured sample at different angles to RD and TD. In the general case of an anisotropic substructure, arrangement of defects and lattice distortion along normals $<\mathrm{hkl}>$ change by passing from one normal of this type to another. As a result, structure-sensitive parameters of the registered X-ray line (hkl) depend on a orientation of the reflecting planes and their normals relative to characteristic directions of the anisotropic substructure.

Grain substructure in rolled metal sheets consists typically of unequiaxed elements being extended often along RD. By this is meant that, in particular, an average distance between neighbouring dislocation subboundaries along RD is larger than along TD, whereas residual lattice distortion along TD is higher than along RD. If, within grains of some texture component, the normal to one family of planes $\{\mathrm{hkl}\}$ is close to RD 
and the normal to other family is close to TD, it should be expected that X-ray reflections from these families would differ by their intensities and half-widths: the first family would give a higher and narrower $\mathrm{X}$-ray line than the second one.

Mutual inequality of texture maxima, corresponding to the same component, is a usual manifestation of substructure anisotropy in metal sheets not only after rolling, but after subsequent annealings as well. Whereas intensities of the X-ray reflection from different families of planes $\{\mathrm{hkl}\}$, registered by texture measurements of rolled samples, decrease due to an anisotropic defect distribution and lattice distortion, an analogous phenomenon can occur also in annealed samples being connected with anisotropic perfection of reflecting grains and resulting anisotropic development of primary extinction (James 1950). Anisotropic lattice perfection by annealing is a consequence of the substructure anisotropy, having been formed initially by rolling, followed by transformation into a structure anisotropy of some new kind. Since rolled and annealed samples differ sharply in their lattice condition and size of perfect substructure elements, their substructure anisotropy manifests itself through different X-ray effects - widening of X-ray line profile and primary extinction, respectively.

The considered phenomenon is rather complicated, therefore for its incontestable demonstration some additional conditions should be met. Worthy of mention are the following ones, though some of them seem to be self-evident:

- in order to eliminate an influence of defocalization on intensities of texture maxima to be compared, these maxima should be equidistant from the centre of DPF, providing, by doing so, the same intensity decrease for both;

- by diffractometric recording of both texture maxima, the irradiated area of the rotating sample should be the same, what is assured by the round form of the sample;

- the studied surface of the sample should be as smooth as possible, without any distinct relief;

- if in consequence of a used annealing both texture maxima of interest changed their intensities, we should be sure that these changes were connected neither with formation of a new recrystallization texture, nor with mutual absorbtion of different components.

All enumerated conditions were satisfied by texture measurements in reference to rolled alloyed molybdenum showing changes of mechanical anisotropy after annealing at the polygonization temperature, which should not be accompanied by texture changes. However, it was revealed that in consequence of anisotropic substructure rearrangement the seeming texture change takes place by polygonization, being responsible for possible errors in the mathematical texture description of the annealed sample.

According to metallographic data, the recrystallization temperature of the studied alloy is near $1300^{\circ} \mathrm{C}$, while X-ray data testify about beginning of its recrystallization by $100^{\circ}$ earlier. Samples for X-ray investigation and mechanical testing were cut from the rolled sheet $0.3 \mathrm{~mm}$ thick and annealed in vacuum at temperatures $1000^{\circ}-1500^{\circ} \mathrm{C}$ for 1 hour. The standard diffractometric method was used to obtain partial DPFs $\{001\}$. In order to follow the course of substructure changes, DPF's were constructed in conventional units of registered intensity, so that primary extinction leads to an overall lowering of intensity levels and can be identified just by this evidence.

In Figure 9 partial DPF's $\{001\}$ are presented for the rolled sample (a) as well as for samples annealed at $1000^{\circ} \mathrm{C}$ (b) and $1200^{\circ} \mathrm{C}$ (c). The rolling texture of the used 

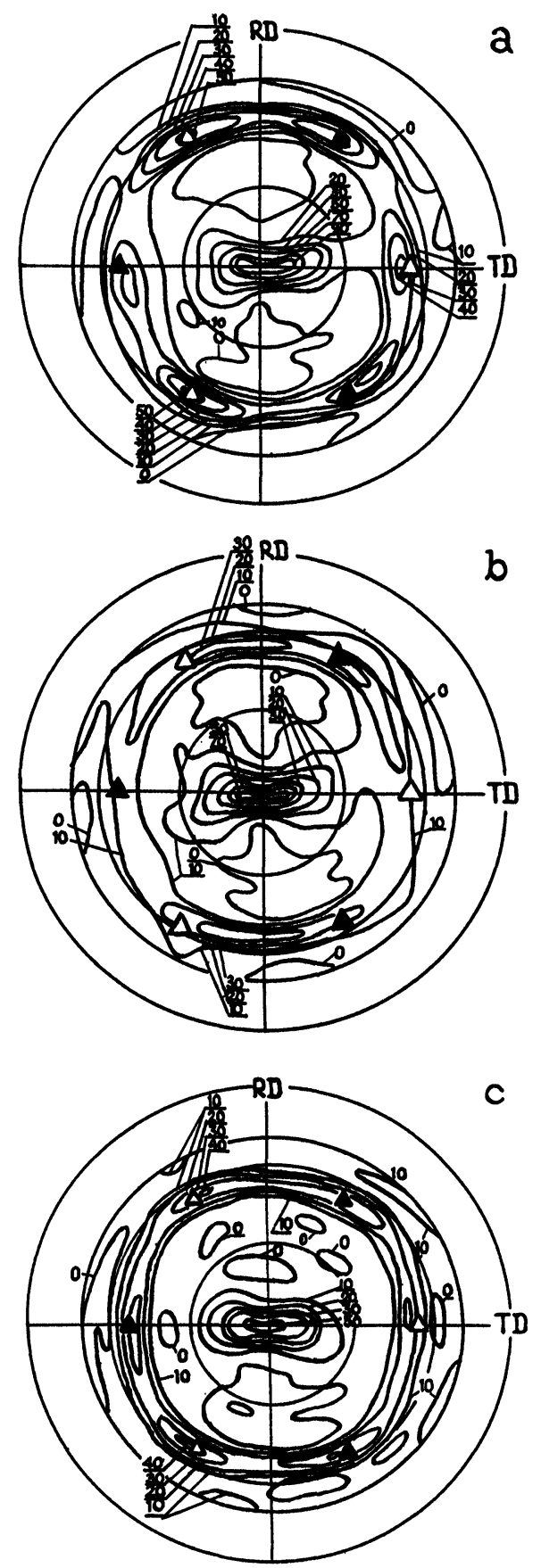

Figure 9 Influence of subrecrystallization annealings on the appearance of DPF $\{011\}$ for sheet samples of alloyed molybdenum:

a - cold rolled, $\varepsilon=70 \% ; \mathrm{b}-$ annealed $1000^{\circ} \mathrm{C}, 1 \mathrm{~h} ; \mathrm{c}-$ annealed $1200^{\circ} \mathrm{C}, 1$ h. $\Delta-(11 \mathrm{i})[1 \overline{1} 0]$, $\Delta$ - (111)[ī10]. 
alloyed molybdenum is quite usual for $\mathrm{BCC}$ metals and contains two separate groups of components with the main ones $\{001\}<011>$ and $\{111\}<011>$. While the single visible maximum of the first component is located at the centre of DPF, all six maxima of both components $\{111\}<011>$ are situated at the circle with an angular radius of $55^{\circ}$. Transition components are only slightly pronounced in this texture. The microstructure of the rolled sheet has a layered character, such that within some regions of successive layers two different stable components develop alternatively. These regions originate from grains with different initial orientations and they differ essentially by strain hardening and tendency to recrystallization. The mobility of high-angle boundaries between regions with different textures is comparatively low owing to microparticles of carbide phases located there preferable. As a result, when the annealing temperature does not exceed $1300^{\circ} \mathrm{C}$, processes of structure perfection, including initial stages of recrystallization, develop within these different regions mutually independently, being localized within the boundaries of one or the other group of close texture components. Therefore, while focussing hereafter upon perfection processes within components $\{111\}<011>$, we can restrict our consideration to the circular zone of DPF $\{001\}$, where maxima of these components are arranged and redistribution of diffraction intensity is confined.

All six maxima of the components $\{111\}<011>$ are equidistant from the centre of DPF, and while four of them are offset by $30^{\circ}$ from the diameter RD-RD, two remaining maxima lie at the diameter TD-TD (Figure 9). Thus, corresponding families of reflecting planes $\{001\}$ as well as normals to them differ sharply by their orientations relative to external axes RD and TD. For simplicity sake these maxima will be denoted as either RD- or TD-maxima depending on their position on DPF $\{001\}$, though in reality normals $<001>$ of interest are deflected from RD or TD by at least $25^{\circ}$. When comparing intensities of RD- and TD-maxima, which should be equal in the case of isotropic substructure, it is possible to estimate semiquantitatevily a degree of substructure anisotropy in grains with orientation $\{111\}<011>$. Plots on Figure 10 show changes of maxima intensities $I_{R D}$ and $I_{T D}$ (a) and their ratio $I_{R D} / I_{T D}$ (b) versus annealing temperature $t^{\circ}$ an. Experimental points corresponding to measured values of maxima intensities fall into zones of scatter shown by hatching. Figure 10-c presents data of mechanical testing by tension (relative elongation $\delta$ ) along RD, TD and at an angle of $45^{\circ}$ to $\mathrm{RD}$ for samples annealed at different temperatures $\mathrm{t}_{\mathrm{an}}^{\circ}$. Since this paper is not an appropriate place for discussion concerning mechanisms of embrittlement in alloyed molybdenum, these data are used only as a visual evidence that subrecrystallization annealings change mechanical anisotropy of the rolled molybdenum sheet in some correlation with mismatched changes of maxima intensities exhibited by components $\{111\}<011>$.

Analysis of the presented data allows to reveal the following experimental facts:

1) A sharp fall of registered intensities $I_{R D}$ and $I_{T D}$ in conditions of lattice perfection by annealing at subrecrystallization temperatures $1000^{\circ}-1150^{\circ} \mathrm{C}$ (Figure 10 -a) has primary extinction as its single reason.

2) Annealing of the rolled sheet at temperatures $1200^{\circ}-1250^{\circ} \mathrm{C}$, corresponding to the initial stage of recrystallization, does not give rise to primary extinction (Figure 10-a), since recrystallization nuclei break the substructure of grains with rolling plane $\{111\}$ and remedy its anisotropy (Figure10-b). 


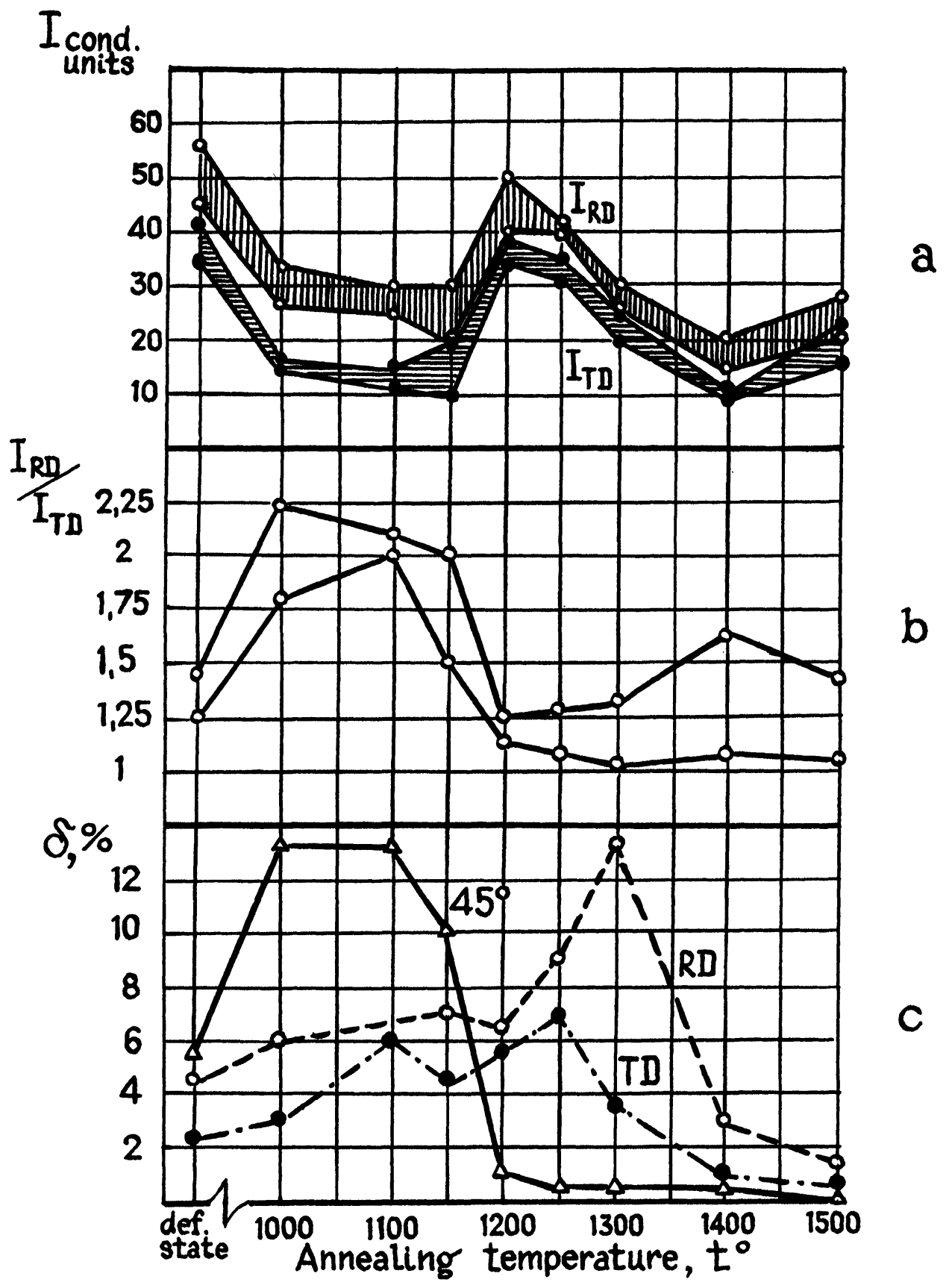

Figure 10 Measured texture parameters and mechanical properties of sheet of alloyed Mo versus temperature of annealing $\mathrm{t}^{\circ}$ an for $1 \mathrm{~h}$ : a - intensity of texture maxima: $\mathrm{I}_{\mathrm{RD}}$ - vertical hatching, $\mathrm{I}_{\mathrm{TD}}$ - horizontal hatching; $\mathrm{b}$ - ratio of texture maxima $\mathrm{I}_{\mathrm{RD}} / \mathrm{I}_{\mathrm{TD}} ; \mathrm{c}$ - relative elongation $\delta$ along, RD, TD and at $45^{\circ}$ to $\mathrm{RD}$. 
3) A significant increase of the ratio $I_{R D} / I_{T D}$ in consequence of subrecrystallization annealings (Figure 10-b) testifies that primary extinction weakens TD-maxima to a greater than RD-maxima; i.e. processes of lattice perfection develop more actively transverse to RD than along it and, as a result, the substructure anisotropy of considered grains builds up.

4) Whereas the components $\{111\}<011>$ are identified uniquely by DPF $\{001\}$ for the rolled sample (Figure 9-a) as well as for the sample annealed at $1200^{\circ} \mathrm{C}$ (Figure 9-c) corresponding to an initial stage of recrystallization, such an identification occurs to be impossible after annealing of the rolled sample at $1000^{\circ} \mathrm{C}$ (Figure 9-b) corresponding to developed polygonization, which can cause only insignificant texture changes.

It should be noted that the intensity redistribution for X-ray reflection (004) observed after annealing at $1000^{\circ} \mathrm{C}$ on DPF $\{001\}$ in limits of the circular zone with a radius of $55^{\circ}$ (Figure 9-b) can not be interpreted as an evidence of some grain reorientation involving a change of the initial rolling plane, because outside of this zone any intensity redistribution has no place. So, the fallacious character of obtained experimental data about texture of the annealed sample is obvious, being connected with anisotropic primary extinction owing to anisotropic lattice perfection. But nevertheless, by a formal approach to texture analysis using ODF technique, this fallacy would be ignored and some imaginary texture components would be found.

The considered case of anisotropic X-ray scattering capacity is rather typical for rolled metal materials, which experienced definite heat treatments.

As for an anisotropy of relative elongation $\delta$, it is important that $45^{\circ}$-elongation $\left(\delta_{45^{\circ}}\right)$ falls down to zero as soon as recrystallization nuclei begin to form in grains of the component $\{111\}<011>$, while the similar decrease of $\delta_{\mathrm{RD}}$ and $\delta_{\mathrm{TD}}$ takes place after annealing at a temperature by $200^{\circ}$ higher, corresponding to recrystallization of the component $\{001\}<011>$ (Figure 10-c). Hence, increased values of $\delta_{45^{\circ}}$ are conditioned by the presence of unrecrystallized components $\{111\}<011>$. In grains of these components tension is directed along crystallographic axes being stable relative to tension, as contrasted to grains of the component $\{001\}<011>$. Under tension along a stable axis a grain orientation remains unchanged as well as misorientation of neighbouring grains. Apparently, these factors are of decisive importance for registered plasticity of molybdenum at the room temperature. Recrystallization of the studied alloyed molybdenum is accompanied by formation of disperse particles of carbide phases and by resulting embrittlement of recrystallized grains (Morgunova, Klipin, Boyarshinov et al. 1975).

\section{CONCLUDING REMARKS}

Above-presented experimental data being obtained in the course of different investigations are gathered within this paper together, since all concern unreliability of $\mathrm{X}$ ray texture measurements because of various physical errors, which can be avoided only by some special conditions. However, in most cases existence of this problem is not realized as a sufficiently serious fact. In particular, an obvious gap arises between recent mathematical elaborations in the field of texture description and actual needs of experimental texture analysis. Wide usage of mathematical procedures for treatment, 
presentation and discussion of texture data relegates, unfortunately, a number of important physical effects to the background. Meanwhile, namely these effects determine the reasonable limits for usage of complicated mathematical procedures in texture analysis.

To take into account considered physical errors of texture measurements is of prime importance for the development of texture analysis as an advantageous method to study various processes of structure formation in metal materials. Such a usage is based on a high sensitivity of texture even to minor alterations in acting mechanisms of structure formation. When these alterations take place only within grains of some orientations, texture analysis appears to be the unique method for their revealing. Therefore, phenomena responsible for physical errors of texture measurements get in the general set of various processes having an effect on texture parameters.

\section{References}

Borodkina M. M and Spektor E. N. (1981). "X-rays analysis of texture in metals and alloys", Metallurgiya, Moscow (in Russian).

Bunge, H. J. (1982). Inhomogene Texturen. Z. Metallkunle, 73, 483-488.

Damask A. C. and Diens G. J. (1963). "Point defects in metals", Gordon and Breach, New York - London.

Douglass D. L. (1971). "The metallurgy of Zirconium", International Atomic Energy Agency, Vienna.

Isaenkova M. G. and Perlovich Yu. A. (1987a). Kinetics and mechanisms of texture formation in $(\alpha-$ $\mathrm{Zr}$ by rolling. Fizika Metallov I Metallovedenie, 64, 107-112 (in Russian).

Isaenkova M. G. and Perlovich Yu. A. (1987b). Reorientation of $\alpha$-Zr crystallites by deformation. Izvestiya Akademii Nauk SSSR, Metalli, 3, 152-155 (in Russian).

James R. W. (1948). "Optical principles of X-ray diffraction", London.

Krivoglaz M. A. (1967). "The theory of X-ray and thermal neutron scattering by real crystals", Nauka, Moscow (in Russian).

Macegorin I. V., Rusakov A. A. and Evstyuchin A. I. (1980). Analysis of the texture formation mechanism in $\alpha-\mathrm{Zr}$ by use of computer modelling. In: Metallurgy and metal science of pure metals N14, edited by V. S. Emelyanov, pp. 39-52, Atomizdat, Moscow (in Russian).

Morgunova N. N., Klipin B. A., Boyarshinov V. A., Tarakanov L. A. and Manegin Yu. V. (1975). "Molybdenum alloys", Metallurgia, Moscow (in Russian).

Perlovich Yu. A. (1983). Inhomogeneity of strain hardening, recovery and recrystallization in molybdenum alloys with a developed crystallographic texture. In Structure, texture and mechanical properties of deformed Molybdenum, edited by V. I. Trefilov, Naukova Dumka, Kiev, 88-145 (in Russian).

Perlovich Yu. A. (1994). Development of strain hardening inhomogeneity during texture formation under rolling of bcc-metals. In Numerical prediction of deformation processes and the behaviour of real materials (Proc. 15th Riso International Symposium on Material Science), edited by S. I. Andersen et al., pp. 445-450, Riso National Laboratory, Roskilde, Denmark.

Tenckhoff E. (1980). "Verformungsmechanismen, Textur und Anisotropie in Zirkonium und Zircaloy", Mat. techn. Reihe, N5, Gebruder Borntrager, Berlin-Stuttgart.

Warren B. E. (1969). "X-ray diffraction", New York.

Wassermann G. and Grewen J. (1962). "Texturen metallischer Werkstoffe", Springer-Verlag, Berlin - Gottingen - Heidelberg. 